\title{
Bilateral field interactions and hemispheric asymmetry in number comparison
}

\author{
Elie Ratinckx*, Marc Brysbaert, Bert Reynvoet \\ Department of Experimental Psychology, Ghent University, Henri Dunantlaan 2, B-9000 Ghent, Belgium \\ Received 12 November 1999; received in revised form 19 September 2000; accepted 20 October 2000
}

\begin{abstract}
We investigated bilateral field interactions and hemispheric asymmetry in number comparison. A numerical comparison task with three different stimulus notations (Arabic numerals, word numerals and bar graphs) was used. In all conditions, a target was displayed in one visual field, simultaneously with a distractor of the same format in the other visual field. Participants had to indicate manually whether the magnitude of the target was small or large, ignoring the distractor stimulus. Only in the condition with Arabic numerals did we obtain some evidence for a LVF advantage, which argues against a strong laterality of number magnitude representations. Significant interactions between target and distractor values were observed, indicating rich interhemispheric interactions. The interactions were mainly situated at the response stage, but the presence of a bilateral identity effect also points to interactions at the input level. (C) 2001 Published by Elsevier Science Ltd.
\end{abstract}

Keywords: Laterality; Interhemispheric interactions; Corpus callosum; Number magnitude

\section{Introduction}

Moyer and Landauer [73] were the first to show the existence of quantitative number representations in humans. In a number comparison task, reaction times decreased systematically when the distance between two numbers increased (see also [35,41]). It was easier to decide that 8 was larger than 2 , than that 8 was larger than 7. The presence of a numerical distance effect seems to indicate that humans convert numbers more or less automatically from a symbolic type of notation to a semantic quantity-based analogical representation.

In the last decade, several cognitive models of number processing have been proposed (e.g. $[20,22,29,34,44,70,71])$ in which magnitude representation plays an important role. For example, in 1992, Dehaene [29] proposed a triple-code model in which an analogical magnitude line is one of the three basic representations. This number line forms the meaning of small integers (see also [18]) and is used for magnitude comparison and approximate calculation. The two

\footnotetext{
* Corresponding author. Tel.: + 32-9-2646441; fax: + 32-92646496.

E-mail address: elie.ratinckx@rug.ac.be (E. Ratinckx).
}

other representations are (a) the visual Arabic numeral form, which represents numbers in Arabic notation and is used for multidigit operations and parity judgment; and (b) the verbal word frame which is used to organize numbers as syntactic sequences of words and which forms the primary code for retrieving learned arithmetic facts [33]. How the different representations are accessed from external stimuli depends on the format in which numbers are presented. As the task proceeds, numbers can be translated via internal transcoding routes in whatever code is required for the task at hand [32].

One of the merits of the triple-code model [29] is that it is neuro-anatomically elaborated. The following neuro-anatomical substrates and connections between the three number representations have been proposed. First, the analogical magnitude representation is assumed to have a copy in the intraparietal sulcus of both hemispheres. Evidence for the bilateral representation of number magnitude can be found in studies with split-brain patients (e.g. [26,81]) in whom both hemispheres are able to compare numbers. The involvement of the intraparietal sulcus in the manipulation of numerical quantities has further been confirmed in several brain-imaging studies [30,37,78]. Second, the visual 
Arabic code is localized in the left and right occipitotemporal areas belonging to the ventral visual pathway dedicated to visual recognition. Neuropsychological evidence for the bilateral representation of the visual Arabic code has been found in several studies (e.g. [24-26,31]). Finally, the verbal code is localized in the classical language areas of the left hemisphere, such as the inferior frontal and superior and middle temporal gyri [32]. Support for the left hemispheric lateralization of the verbal code can be found in studies with splitbrains (e.g. [26,46]) and patients with left hemispheric lesions (e.g. [31,38]).

Both copies of the analogical magnitude representation and the visual Arabic number form are assumed to be connected via direct transcallosal pathways. The model does not specify a hemispheric dominance of the Arabic or magnitude representations, but leaves open the possibility of some degree of differentiation between homologous left and right areas, such as right-hemispheric dominance for the representation of continuous numerical quantities (e.g. [2,65]). Indeed, some data suggest that larger-smaller comparison may be more affected by right-hemisphere lesions than by left-hemisphere lesions $[28,80]$. In contrast, other neuropsychological data suggest that the quantity representations of the left and right hemispheres may have very similar characteristics. In a digit comparison task, a split-brain patient showed the same numerical distance effect in both hemispheres [26]. In the same study an overall right visual field (RVF) advantage in digit comparison was found.

\subsection{Lateralization studies in number processing}

Visual half field (VHF) studies of number processing have used different kinds of numerical tasks such as number recognition (see Table 1a for reaction time data and Table $1 \mathrm{~b}$ for accuracy data), odd-even judgment (Table 2), and magnitude evaluation (Table 3), as well as several types of stimulus formats (dot clusters, bar graphs, word numerals and digits). Boles [7] is the only study that crossed different types of stimulus materials with different types of judgment to find out whether VHF differences in number processing depend primarily upon the format in which the numerical information is presented, or upon the kind of task participants have to perform. On the basis of his results, Boles concluded that VHF advantages in number processing depend upon the format of the numerical display only: Word numerals yielded a RVF superiority, whereas dot clusters and bar graphs yielded a left visual field (LVF) advantage, irrespective of the task. Digits did not give rise to a VHF advantage (see also [8]; experiment 3). A closer look at the data, however, revealed a trend for smaller VHF differences in magnitude judgment (a 3 ms RVF advantage with dot clusters, bargraphs, and dials) than in odd-even judgment (a 38 ms LVF advantage) or number recognition (a $36 \mathrm{~ms}$ LVF advantage; see ([7]; experiment 1)). So, Boles's input interpretation of VHF differences in numerical cognition may have been overstated. Unfortunately, Boles [7] did not provide individual data for the different cells of his

Table 1a

VHF differences in the recognition/enumeration of Arabic digits, word numerals, dot clusters and bar graphs (RTs in ms)

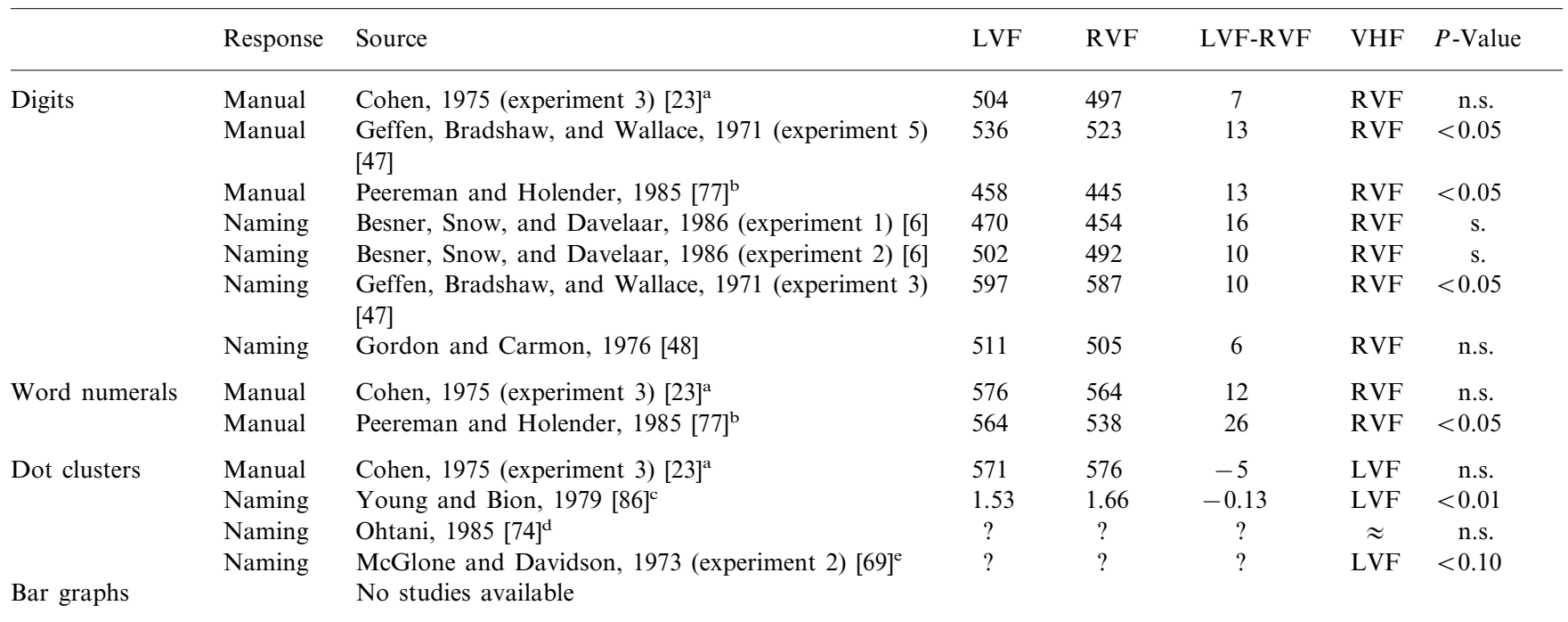

a Averaged across cued/uncued conditions.

${ }^{\mathrm{b}}$ Only RTs on numbers in the number-non-number classification task are included.

${ }^{\mathrm{c}} \mathrm{RTs}$ are averaged across age and gender; RTs are presented in seconds.

${ }^{\mathrm{d}}$ LVF advantage $(P<0.01)$ when error trials are included in the analysis.

e Results are frequency scores.

${ }^{\mathrm{f}}$ Note: Studies with lesion patients $[61,82,84]$ also suggest a right hemisphere dominance in dot enumeration. 
Table $1 b$

VHF differences in the recognition/enumeration of Arabic digits, word numerals, dot clusters and bar graphs (percentage of correct recognition) ${ }^{\mathrm{c}}$

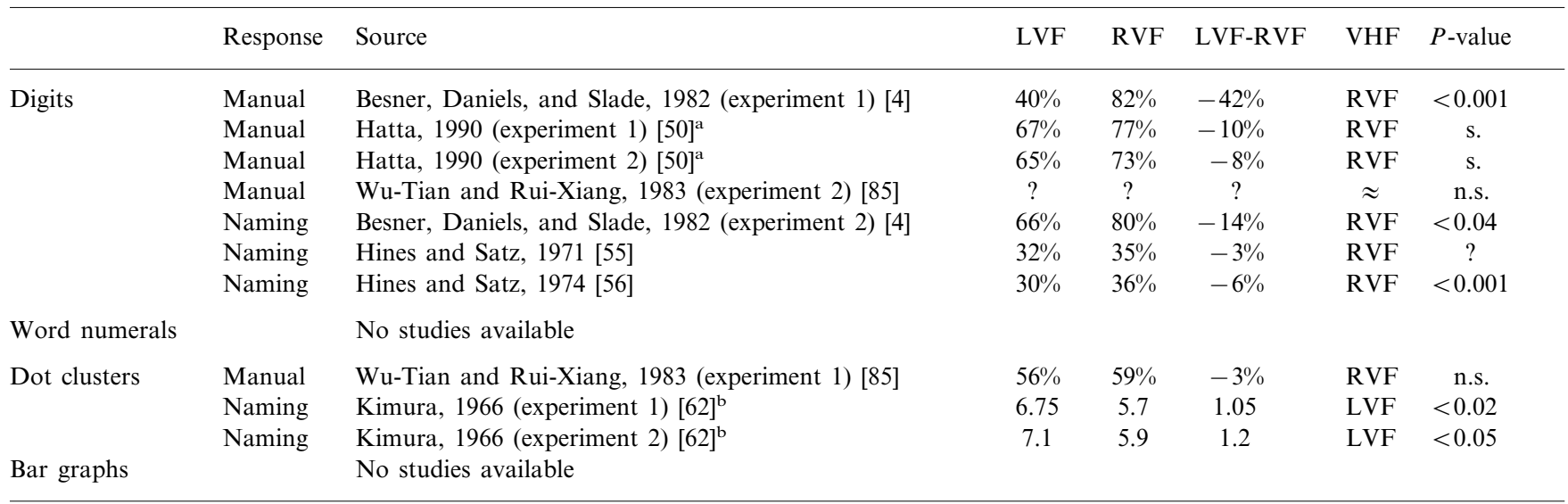

\footnotetext{
a Participants are young children of 4,5 and 6 years old.

${ }^{\mathrm{b}}$ Values are mean accuracy scores showing a left visual field advantage.

${ }^{\mathrm{c}}$ Note: Studies with multidigits are not presented in the table: However, most studies produce RVF advantages ([12,21,40,51]; experiment 1), while one study finds no difference [60]. Another study [57] observes a LVF advantage with free order of recall of digits and a RVF advantage with fixed order of recall.
}

design (see also [8]; experiment 3), so that this study could not be retained in the reviews of Tables $1 \mathrm{a}, 1 \mathrm{~b}, 2$ and 3 .

Although the picture is suboptimal for some cells of the design formed by stimulus type $x$ judgment type, laterality research on number processing suggests the following general lines (see Tables 1a, 1b, 2 and 3). The judgment of word numerals consistently produces RVF advantages in number recognition and odd-even judgment, but not in magnitude judgment (one study only), where a null effect was observed. Dot clusters and bar graphs nearly always give rise to a LVF advantage in number recognition and odd-even judgment, but again fail to evoke a significant VHF advantage in the single study that looked at magnitude judgment. Finally, Arabic numerals thus far yielded the most inconsistent pattern of results. When the participant's task is to recognize digits, RVF advantages are the rule. However, magnitude and odd/even judgment tasks elicit the whole range of effects, going from LVF superiorities to RVF superiorities and null effects. To the data of Tables 1a, 1b, 2 and 3, those of Boles ([7], experiment 2) must be added. This study reported a non-significant $11 \mathrm{~ms}$ LVF advantage for Arabic digits (averaged across number recognition, magnitude judgment, and parity judgment), against a significant $19 \mathrm{~ms}$ RVF for word numerals (averaged over the same tasks).

All in all, VHF differences in number processing seem to be a function of both stimulus characteristics and task requirements. Word numerals usually elicit a RVF advantage, except for magnitude estimation. Bar graphs and dot clusters predominantly produce a LVF advantage, but not in magnitude estimation. Arabic numerals mostly give rise to a RVF advantage in number recognition, but there is little unequivocal evi- dence for hemispheric specialization of Arabic numerals in odd-even judgment and magnitude judgment. A small caveat to this general picture is that the number of studies about magnitude judgment may be too small to draw firm conclusions for this particular task, even though the absence of a clear VHF asymmetry agrees with Dehaene's [29] triple-code model of number processing, which says that the analogical number magnitude system is used for magnitude judgment and is bilaterally represented.

\subsection{Interhemispheric interactions in number processing}

Only a few studies have examined interhemispheric interactions in number processing. Lambert [67] presented a bilateral variant of the negative priming paradigm to 2 split-brain patients and 7 neurologically intact participants. A target digit was presented in RVF simultaneously with a distractor digit in the LVF. The task was to categorize the target number as odd or even. For both normals and split-brains, a significant negative priming effect was found (i.e. responses were slower when the target was the distractor of the previous trial than when the target was unrelated to the distractor of the previous trial). To explain the interhemispheric effect in split-brains, Lambert suggested a mediation of yet poorly understood non-callosal pathways linking cortical and mid-brain areas. Previously, Lambert [66] had shown a similar interhemispheric exchange of semantic verbal information in split-brains (see also [27]). Next to the negative priming effect, Lambert [67] also reported a non-significant response compatibility effect (i.e. for the normal participants responses were $17 \mathrm{~ms}$ faster when both target and distractor were odd or even than when one was odd 
and the other even or vice versa; the same was true for one of the split-brains; the other showed a non-significant trend in the opposite direction).

Other studies have examined interhemispheric collaboration during mental addition in normal adults. The common factor in these experiments was the addition of two numbers that were presented either unilaterally in LVF or RVF, or bilaterally. Two studies [52,53] reported a significant bilateral field advantage, suggesting that the processing power of the brain is enhanced when interhemispheric collaboration is encouraged. One study [3], however, failed to show this advantage of bilateral processing over unilateral processing. Previously, Dimond and Beaumont [40] had already observed that the recall of two digits was better with bilateral presentation than with unilateral presentation.

\section{The present study}

The present study was set up with two goals in mind. First, we wanted to verify whether VHF differences are indeed absent in the magnitude judgment task. As indicated above, this task has been investigated the least despite its core role in Dehaene's [29] triple-code model. Second, we wanted to evaluate the assumption of the triple-code model that the left and right magnitude representations are strongly intertwined through interhemispheric connections. More specifically, we tested whether interhemispheric interactions are located at early stages such as number identification and access to the semantic number line, or at the level of the motor response, or both.

To test these issues, we ran three manual reaction time experiments, with three different presentation formats (Arabic numerals, word numerals and bar graphs). The participant's task was to classify a target number as small (1 and 2) or large (5 and 6). We used a variant of Lambert's [67] interhemispheric Stroop task. The target stimulus was presented randomly in RVF or LVF, together with a distractor in the opposite VHF. Participants were asked to react to the target number and to ignore the distractor. This technique with bilateral stimulus display not only allowed us to look for interhemispheric interactions, but is also recommended by several authors as the best way to show

Table 2

VHF differences in the odd/even judgment of Arabic digits, word numerals, dot clusters and bar graphs (RTs in ms)

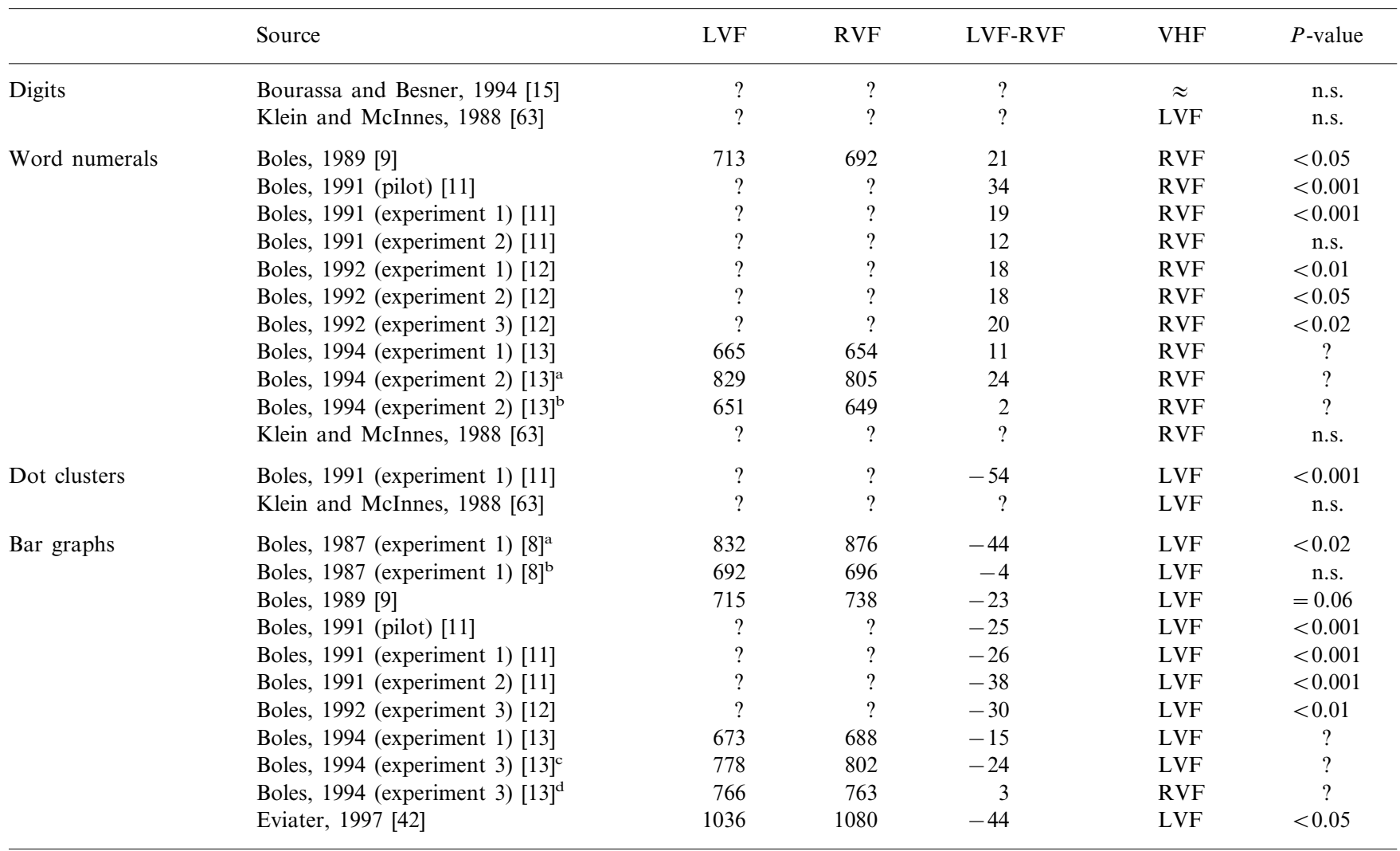

a Bilateral display.

${ }^{\mathrm{b}}$ Unilateral display.

${ }^{\mathrm{c}}$ High eccentricity.

${ }^{\mathrm{d}}$ Low eccentricity. 
Table 3

VHF differences in the magnitude judgment of Arabic digits, word numerals, dot clusters and bar graphs (RTs in ms)

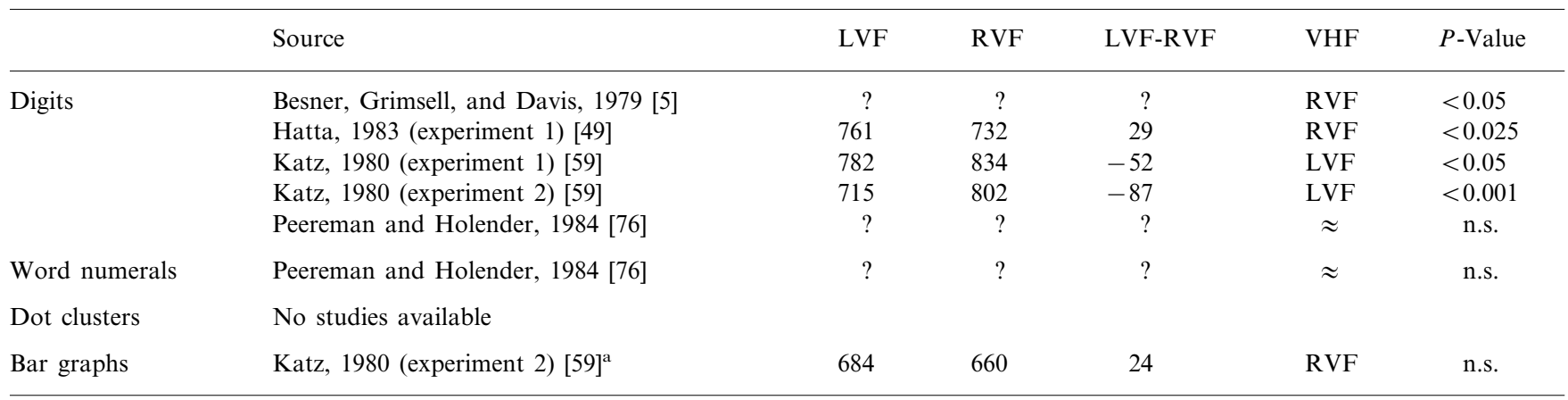

${ }^{a}$ Bar graphs are dots in colums.

behavioral evidence for cerebral asymmetries. In a series of studies, Boles $[7,10,13,14]$ showed that bilateral stimulus displays increase the size of VHF asymmetries (both LVF and RVF advantages) relative to unilateral displays, probably because of the interhemispheric competition induced by the two stimuli. Hellige and Sergent [54] recommended the technique as a way to avoid attentional biases to one or the other VHF.

Our design allowed us to distinguish between interhemispheric interactions at the level of the output and interactions at the level of the input, which will be referred to respectively as response compatibility and quantity priming. To assess response compatibility, we compared trials in which the target and distractor elicited the same response (congruent trials) with trials in which target and distractor required different responses (incongruent trials). Recent studies have shown that small integers in a magnitude judgment task are categorized automatically as larger or smaller than the criterion number and activate the appropriate response [36,64]. An interhemispheric response compatibility effect would show that motor information associated with the number comparison task is integrated across the hemispheres. Quantity priming was evaluated by looking at a decrease in reaction times as a function of the numerical distance between target and distractor. Den Heyer and Briand [39] showed that RTs to numerals are faster when immediately before a prime was shown with a close magnitude (distance of 1) than when a prime was shown with a more distant quantity (see also $[18,64,79]$ ). Such an effect suggests that the prime has activated a semantic representation and that activation spreads to the nearby representations. The existence of an interhemispheric quantity priming effect would suggest that left and right magnitude representations are interconnected.

\section{Method}

\subsection{Participants}

Thirty-six undergraduates (12 in each experiment) of Ghent University participated for course credit. All participants (age range $=18-32$ years) were male and right-handed as confirmed by a Dutch translation of the Oldfield [75] questionnaire. Participants were unaware of the purpose of the experiment and had normal or corrected-to-normal vision.

\subsection{Procedures common to all experiments}

Stimuli were presented on a Pentium PC connected to a 15' colour Yakumo monitor. Responses were measured with an external four-key board connected to the gameport of the PC. Stimulus presentation and response timing were measured to the nearest millisecond with the software routines of Brysbaert and colleagues $[16,17,19]$.

The fixation stimulus was made of two short vertical lines of $0.4^{\circ}$, one above the other, separated by a gap of $0.6^{\circ}$. A stimulus display consisted of a target stimulus presented in one VHF, an arrow presented in the gap of the fixation mark and pointing to the target, and a distractor stimulus presented in the opposite VHF. A trial started with a foreperiod of $1000 \mathrm{~ms}$, followed by the presentation of the fixation stimulus, a second foreperiod of $500 \mathrm{~ms}$, and the stimulus display shown for $100 \mathrm{~ms}$.

The participants were instructed to fixate the gap of the fixation mark and to judge, as quickly and as accurately as possible, the magnitude of the target indicated by the central arrow. Magnitudes were small if they were 1 or 2 , and large if they were 5 or 6 . The participants sat at a distance of about $60 \mathrm{~cm}$ from the 
screen (there were no head restraints). At a distance of $57 \mathrm{~cm}$, stimulus magnitude of $10 \mathrm{~mm}$ corresponds with a visual angle of $1^{\circ}$.

Participants' responses were bimanual in order to avoid stimulus-response compatibility effects. The response box consisted of four buttons, arranged in such a way that they could easily be pressed with digits and middle fingers (i.e. the two outer buttons were placed $15 \mathrm{~mm}$ above the inner buttons). Half of the participants were instructed to press the two upper buttons simultaneously with the middle finger of the right and the left hand when the target was large, and to press the lower buttons with the digits when the target was small. The other half of the participants was given the opposite stimulus-response assignment. Participants were instructed to ignore the distractor stimulus.

Each participant completed a training block of 32 trials and four experimental blocks of 128 trials. This resulted in a total of 16 observations per participant in each of the $2 \times 4 \times 4$ (VHF $\times$ Target Value $\times$ Distractor Value) $=32$ within-subjects conditions. At the end of both the practice and experimental sessions the participants received feedback about their RTs and accuracy.

\subsection{Experiment 1}

Stimuli were the Arabic numerals 1, 2, 5 and 6, extending $0.3 \times 0.4^{\circ}$ horizontally and vertically. Both targets and distractors were presented $1.3^{\circ}$ left or right of the fixation mark (measured from the center of the stimulus to the vertical line of the fixation mark).

\subsection{Experiment 2}

Stimuli were the Dutch word numerals 'een', 'twee', 'vijf', and 'zes', printed in lower case. The words were presented horizontally and extended $1-1.3^{\circ}$ horizontally and $0.5^{\circ}$ vertically. Both targets and distractors were presented at $0.8^{\circ}$ left or right of the fixation mark (measured from the vertical line of the fixation mark to the nearest edge of the first (RVF) or the last letter (LVF) of the word).

\subsection{Experiment 3}

Bar graphs comprised of a rectangular vertical bar, with the vertical length of the bar denoting a numerical magnitude of $1,2,5$ or 6 . Unlabelled horizontal reference lines (going from $0.1^{\circ}$ to the left of the bar to $0.1^{\circ}$ to the right of the bar) were displayed at the $0,2,4$ and 6 level. A bar graph display denoting a magnitude of 6 was $0.8^{\circ} \times 2.3^{\circ}$ in extent. Both targets and distractors were presented at $1.3^{\circ}$ left or right of the fixation mark (measured from the center of the stimulus to the vertical line of the fixation mark).

\section{Results}

Only average reaction times (RTs) of correct responses in the range of $150-1500 \mathrm{~ms}$ were analyzed. In all three experiments, error rate did not exceed 19.1\% per participant (average $=8.4 \%$ ). In addition, the percentage of outliers, averaged across participants, amounted to only $0.58 \%$ of the correct responses. There was no speed-accuracy trade-off, as indicated by a positive correlation between RT and number of errors over the 32 cells of the design (Arabic numerals: $r=+$ $0.78, n=32, P<0.05$; Word numerals: $r=+0.65, n=$ 32, $P<0.05$; Bar graphs: $r=+0.48, n=32, P<0.05$ ).

\subsection{Analysis of variance}

A separate $2 \times 4 \times 4$ ANOVA was performed for each experiment with the following within-subjects factors: VHF (2 levels), Target Value and Distractor Value (4 levels each).

The main effect of VHF was marginally significant in Experiment 1 (Arabic digits) $(\mathrm{LVF}=623 \mathrm{~ms} ; \mathrm{RVF}=$ $643 \mathrm{~ms} ; F(1,11)=5.02, \mathrm{MSE}=7120.2, P=0.047)$, due to a LVF advantage. There was a tendency towards a $\mathrm{LVF}$ advantage for bar graphs $(\mathrm{LVF}=605 \mathrm{~ms}$; $\mathrm{RVF}=$ $610 \mathrm{~ms} ; F<1.8)$, whereas for word numerals $(\mathrm{LVF}=$ $659 \mathrm{~ms} ; \mathrm{RVF}=658 \mathrm{~ms} ; \quad F<1)$ no difference was observed.

There was a significant main effect of Target Value in word numerals (Value $1=625,2=693,5=653,6=$ $663 \mathrm{~ms} ; F(3,33)=12.73, \quad \mathrm{MSE}=5903.7, P<0.0001)$ and in bar graphs (Value $1=587,2=628,5=622,6=$ $592 \mathrm{~ms} ; F(3,33)=6.21, \mathrm{MSE}=6756.6, P<0.005)$. The effect in bar graphs is probably due to a numerical distance effect (see [73]): The most extreme values (1 and 6) produced the fastest reaction times as revealed by post hoc comparisons (Tukey; $P<0.05$ ). The difference between the values 6 and 5 did not reach significance $(P=0.07)$. The same distance effect was present in Arabic digits but failed to reach significance (Value $1=616,2=634,5=643,6=638 \mathrm{~ms} ; \quad F=2.7 ; \quad P=$ 0.06). Post hoc comparisons (Tukey) for the word numerals revealed that reaction times to the word 'one' were reliably faster than to 'two' or 'six' $(P<0.05)$. This effect may be due to the high frequency of the word 'een' in Dutch ('een' is also the indefinite article 'a' in Dutch).

Finally, there were significant main effects of Distractor Value in the word experiment (Value $1=643,2=662,5=664,6=665 \mathrm{~ms} ; \quad F(3,33)=4.43$, $\mathrm{MSE}=2355.3, P<0.02)$ and in the bar graph experiment (Value $1=625,2=608,5=592,6=604 \mathrm{~ms}$; $F(3,33)=10.23, \mathrm{MSE}=1729.8, P<0.0001)$. Post hoc comparisons (Tukey) showed that reaction times to the word numeral 'one' were significantly faster than to 'five' or 'six' $(P<0.05)$. The effect for bar graphs was 


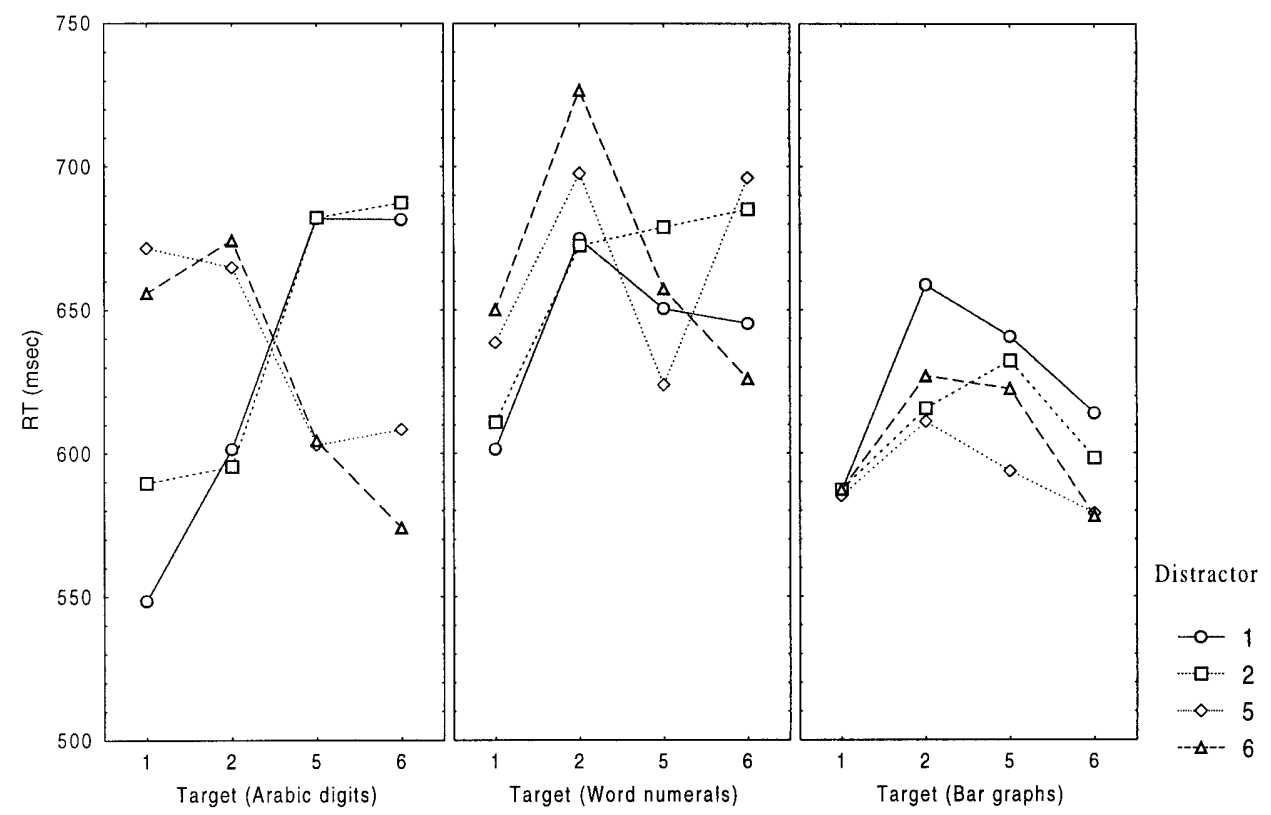

Fig. 1. Two-way interaction between Target Value (on the $x$-axis) and Distractor Value (line pattern) for Arabic digits (a), word numerals (b), and bar graphs (c), respectively.

due to the fact that RTs to the value 1 were significantly slower than to all other values (Tukey; $P<0.05$ ). It is not clear how to interpret this observation. The effect in Arabic digits did not reach significance (Value $1=628,2=639,5=637,6=627 \mathrm{~ms} ; F<1$ ).

Significant interactions between Target and Distractor Value were observed both for Arabic digits $(F(9,99)=26.07, \mathrm{MSE}=3079.8, P<0.0001)$ and word numerals $(F(9,99)=7.03, \mathrm{MSE}=2657.5, P<0.0001)$, indicating interhemispheric interactions (see Fig. 1a and b). The bilateral field interaction effect did not reach significance in the experiment with bar graphs (see Fig. $1 \mathrm{c} ; F=1.7)$.

Two interaction effects were observed involving the factor VHF. First, in experiment 1, the two-way interaction between Target and Distractor Value interacted with VHF $(F(9,99)=2.3, \quad \operatorname{MSE}=1730.2, \quad P<0.03)$. Second, for word numerals, a two-way interaction between VHF and Distractor Value was observed $(F(3,33)=2.9$, MSE $=1490.1, P<0.05)$, because participants reacted faster when an odd distractor was presented in LVF (648 ms) than in RVF (659 ms), whereas the reverse was true for even distractors $(669$ vs. $658 \mathrm{~ms}$ ). A preference for the associations left/odd and right/even has been reported in other studies as well (e.g. [79]).

\subsection{Regression analysis}

To test whether the interaction between Target and Distractor Value was due to response and/or quantity priming, a regression analysis for repeated-measures data was run ([68]; method 3; see also [43] for a more extended discussion). Four predictor variables were included: VHF (LVF was coded as -1 and RVF as $+1)$, Absolute Distance between target and distractor $(0,1,3,4$ and 5), Response Compatibility between target and distractor (coded as -1 in the case of a congruent response and as +1 otherwise), and the interaction between VHF and Response Compatibility to evaluate whether response compatibility depended on VHF. The analysis was done for all three experiments (see Table 4).

Besides a significant LVF advantage of $20 \mathrm{~ms}$ for Arabic digits (regression weight $=9.76 ; t(11)=2.33$, $\mathrm{SD}=14.50, P<0.05)$, which repeats the effect previously reported in the analysis of variance, only the effect of Response Compatibility was significant. This effect points to interhemispheric interactions at the level of the response. In experiment 1 (Arabic digits), congruent responses were $64 \mathrm{~ms}$ faster than incongruent responses $\quad$ (regression weight $=32.18 ; \quad t(11)=3.35$, $\mathrm{SD}=33.25, P<0.01)$. Congruent responses were also $38 \mathrm{~ms}$ faster for word numerals (regression weight $=$

Table 4

Regression weights of the model with VHF, distance, response compatibility (RC) and the interaction between VHF and response compatibility $(\mathrm{VHF} \times \mathrm{RC})$

\begin{tabular}{lccc}
\hline & Arabic digits & Word numerals & Bar graphs \\
\hline VHF & $9.76^{*}$ & -0.75 & 3.02 \\
Distance & 5.76 & -3.58 & -1.34 \\
RC & $32.18^{*}$ & $19.18^{*}$ & 7.14 \\
VHF $\times$ RC & 4.90 & 4.82 & -0.13 \\
\hline
\end{tabular}

\footnotetext{
$* \mathrm{P}<0.05$
} 
Table 5

Regression weights of the model with VHF, identity priming (IP) and the interaction between VHF and identity priming $(\mathrm{VHF} \times \mathrm{IP})$

\begin{tabular}{lccc}
\hline & Arabic digits & Word numerals & Bar graphs \\
\hline VHF & 4.85 & -5.44 & 3.00 \\
IP & $10.26^{*}$ & $14.59^{*}$ & $8.66^{*}$ \\
VHF $\times$ IP & 3.28 & 3.82 & -0.62 \\
\hline
\end{tabular}

$* P<0.05$.

$19.18 ; t(11)=3.86, \mathrm{SD}=17.19, P<0.005)$, while the 14 $\mathrm{ms}$ advantage for congruent responses with bar graphs was not significant (regression weight $=7.14 ; t=1.26$ ). The difference in Response Compatibility effect between the different stimulus notations was next to significant $(F(2,33)=3.16, \mathrm{MSE}=595.6, P<0.06)$. In none of the experiments did we obtain a significant effect of Distance, which suggests an absence of interhemispheric transfer at the input level (quantity priming). A regression analysis without the predictor Absolute Distance revealed the same significant effects.

In the previous analysis, the quantity priming effect may have been obscured by the facts (a) that distance and response compatibility were correlated; and (b) that with tachistoscopic presentation times priming effects are often limited to a small range of values around the target [79]. Therefore, we ran another regression analysis on a limited data set, consisting of the trials with congruent responses only (i.e. both numbers were either small or large). The predictor variables included in this analysis were VHF ( LVF coded as -1 and RVF as +1 ), Distance (coded as -1 when both numbers were the same, and +1 in the case of a distance of 1 ), and the interaction between VHF and Distance (see Table 5). The Distance effect was significant for all three notations (Arabic digits: regression weight $=$ $10.26 ; t(11)=2.85, \mathrm{SD}=12.47, P<0.05$; word numerals: regression weight $=14.59 ; t(11)=3.11, \mathrm{SD}=16.24$, $P<0.01$; bar graphs: regression weight $=8.66 ; t(11)=$ $2.81, \mathrm{SD}=10.68, P<0.05)$, indicating faster responses when target and distractor were identical. There was no significant difference in Distance effect between the different stimulus formats $(F(2,33)<1)$. No other effect reached significance.

\section{General discussion}

The research questions of the present study were straightforward. We examined the lateralization of number magnitude representations by looking at VHF differences in neurologically intact persons. We also investigated the presence of interhemispheric interactions in numerical processing by inspecting bilateral field interactions between a target and a distractor.
More specifically, we tested whether the interhemispheric interactions were situated at early stages of processing such as stimulus identification and semantic access, or at late stages such as the motor response, or at both.

Although bilateral stimulus displays have been reported to increase the power to detect VHF differences (e.g. $[7,10,14])$, none of our experiments provided evidence for strong VHF differences in number comparison. Only Arabic digits resulted in a marginally significant LVF advantage of $20 \mathrm{~ms}$. This is in line with two experiments of Katz ([59]; see Table 3) and one experiment of Boles [7], but not with two other studies that reported a RVF advantage [5,49], and one that reported a null effect [76]. In line with Peereman and Holender [76], we found no evidence at all for a main effect of VHF with words. Finally, there was a tendency towards a LVF advantage for bar graphs, but this effect did not reach significance and must be put next to the non-significant RVF advantage reported by Katz [59]. In addition to the absence of a clear VHF main effect, we also failed to find convincing evidence for an asymmetry in the interactions between the left and the right hemisphere. As shown in Table 4 and Table 5, response and quantity priming did not differ as a function of whether the distractor was presented in LVF or RVF. Moreover, the distance effects for bar graphs and Arabic digits as revealed by the main effect of Target Value did not interact with VHF (see also [26]). All these findings point to more or less equivalent access to quantity information in the left and the right cerebral hemisphere. They also corroborate the existing literature (see Tables 1a, 1b, 2 and 3) that magnitude comparison differs in hemispheric terms from oddeven judgment and number recognition, perhaps because magnitude judgment de-emphasizes the perception of specific number information, thereby reducing the visuo-spatial or verbal emphasis of the task [7].

Contrary to the meager evidence for cerebral asymmetries in magnitude judgment, we found clear indications of VHF interactions. As mentioned in the Introduction, such interactions have been reported before $[40,52,53,67]$. However, none of these studies tried to pin down the stages at which the interactions happened. For instance, it could be that the VHF interaction between target and distractor is entirely due to response competition. In such a model, both numbers would be processed separately and in parallel by the left and the right cerebral hemisphere up to the response code associated with each stimulus (in our experiments, pressing with the digits or the middle fingers). Such a model is not infeasible as it is becoming increasingly clear that stimuli which are repeated a number of times and which always require the same response, automatically activate an episodic event-file, which contains the 
action to be performed $([36,58,64]$; for models of parallel information processing in the cerebral hemispheres, see e.g. $[1,45,72,83])$. And indeed, as shown in Table 4, the greater part of the VHF interaction was due to those cases in which the target and the distractor elicited different responses. Interestingly, the time cost of response incongruity seemed to differ between the notations we used, suggesting that the stimulus-response association in the event-file was stronger for Arabic digits than for word numerals, and bar graphs.

To test whether the VHF interaction was entirely due to response incongruity at the final processing stages, we examined whether RTs were faster when target and distractor were the same number than when they were different numbers associated with the same response. The absence of such a quantity priming effect would be strong evidence for independent number processing in the left and the right hemisphere during the initial stages of stimulus perception and semantic access. However, as can be seen in Table 5, for all notations there was a significant bilateral identity priming effect. In addition, the priming effect was of the same magnitude for all stimulus modalities, strongly suggesting that interhemispheric cross-talk happened at a stage involving abstract, modality independent representations. In most models, this stage of numerical processing is thought to be the semantic magnitude system (e.g. $[29,44,70])$.

In conclusion, our data show the absence of strong hemispheric differences in the magnitude judgment task and stress the significance of interhemispheric interactions. The results are in line with Dehaene's [29] neuroanatomical model of number processing, which assumes that the Analogical Magnitude Representation has a copy in the left and right hemisphere and that both copies are linked via direct transcallosal pathways. Our data indicate that the left and right hemispheres are more or less equal in processing magnitude information. The bilateral processing of number magnitude information is in agreement with most other VHF studies on magnitude evaluation (see Table 3) and one split-brain study in which the same distance effect was found in both hemispheres [26]. Our data are also in line with the mixed evidence that comes from research with patients, in which it has been claimed that smaller-larger comparisons seem to be more affected by right-hemispheric than by left-hemispheric lesions $[28,80]$, while at the same time a significant RVF advantage has been reported in a split-brain patient [26]. Our data also indicate the presence of rich interhemispheric interactions. Some of these interactions happened at the later processing stages of response preparation, but there was also evidence for interactions at the level of a notation-independent magnitude system.

\section{Acknowledgements}

The authors thank Wim Fias and two anonymous reviewers for their helpful comments on an earlier draft of the manuscript. They also thank Antoine Tavernier for his technical support. Bert Reynvoet is a research assistant of the Fund for Scientific Research (Flanders, Belgium).

\section{References}

[1] Allen M. Models of hemispheric specialization. Psychological Bulletin 1983;93:73-104.

[2] Baciu M, Koenig O, Vernier MP, Bedoin N, Rubin C, Segebarth C. Categorical and coordinate spatial relations: fMRI evidence for hemispheric specialization. Neuroreport 1999;10:1373-8.

[3] Berger JM, Landolt M. Bilateral processing of numbers: parallel processing versus functional unity. Cortex 1990;26:55566.

[4] Besner D, Daniels S, Slade C. Ideogram reading and right hemisphere language. British Journal of Psychology 1982;73:218.

[5] Besner D, Grimsell D, Davis R. The mind's eye and the comparative judgment of number. Neuropsychologia 1979;17:373-80.

[6] Besner D, Snow D, Davelaar E. Logographic reading: is the right hemisphere special? Canadian Journal of Psychology 1986;40:45-53.

[7] Boles DB. Hemispheric differences in the judgment of number. Neuropsychologia 1986;24:511-9.

[8] Boles DB. Reaction time asymmetry through bilateral versus unilateral stimulus presentation. Brain and Cognition 1987;6:321-33.

[9] Boles DB. Do visual field asymmetries intercorrolate? Neuropsychologia 1989;27:697-704.

[10] Boles DB. What bilateral displays do. Brain and Cognition 1990; 12:205-28.

[11] Boles DB. Factor analysis and the cerebral hemispheres: pilot study and parietal functions. Neuropsychologia 1991;29:5991.

[12] Boles DB. Factor analysis and the cerebral hemispheres: temporal, occipital and frontal functions. Neuropsychologia 1992;30:963-88.

[13] Boles DB. An experimental comparison of stimulus type, display type, and input variable contributions to visual field asymmetry. Brain and Cognition 1994;24:184-97.

[14] Boles DB. Parameters of the bilateral effect. In: Kitterlee FL, editor. Hemispheric communication: mechanisms and models. Hillsdale, NJ: Erlbaum, 1995:211-30.

[15] Bourassa D, Besner D. Semantics at a glance: is the right hemisphere special? Canadian Journal of Experimental Psychology 1994;48:543-52.

[16] Bovens N, Brysbaert M. IBM PC/XT/AT and PS/2 Turbo Pascal timing with extended resolution. Behavior-ResearchMethods-Instruments-and-Computers 1990;22:332-4.

[17] Brysbaert M. A warning about millisecond timing in Turbo Pascal. Behavior-Research-Methods-Instruments-and-Computers 1990;56:479-90.

[18] Brysbaert M. Arabic number reading: on the nature of the numerical scale and the origin of phonological recoding. Journal of Experimental Psychology: General 1995;124:434-52.

[19] Brysbaert M, Bovens N, d'Ydewalle G, Van Calster J. Turbo pascal timing routines for the IBM microcomputer family. Behavior-Research-Methods-Instruments-and-Computers 1989;21:73-83. 
[20] Campbell JID, Clark JM. An encoding complex view of cognitive number processing: Comment on McCloskey, Sokol, and Goodman (1989). Journal of Experimental Psychology: General 1988;117:204-14.

[21] Carmon A, Nachshon I, Starinsky R. Developmental aspects of visual hemifield differences in perception of verbal material. Brain and Language 1976;3:463-9.

[22] Cipolotti L, Butterworth B. Toward a multiroute model of number processing: impaired number transcoding with preserved calculation skills. Journal of Experimental Psychology: General 1995;124:375-90.

[23] Cohen G. Hemisphere differences in the effects of cuing in visual recognition tasks. Journal of Experimental Psychology: Human Perception and Performance 1975;1:366-73.

[24] Cohen L, Dehaene S. La lecture des nombres dans l'alexia pure: effets de la tache et de la specialisation hemispherique. RevueNeurologique 1995a;15:480-5.

[25] Cohen L, Dehaene S. Number processing in pure alexia: the effect of hemispheric asymmetries and task demands. Neurocase $1995 b ; 1: 121-37$

[26] Cohen L, Dehaene S. Cerebral networks for number processing: evidence from a case of posterior callosal lesion. Neurocase 1996;2:155-73.

[27] David AS. Stroop effects within and between the cerebral hemispheres: studies in normals and acallosals. Neuropsychologia 1992;30:161-75.

[28] Dahmen W, Hartje W, Buessing A, Sturm W. Disorders of calculation in aphasic patients spatial and verbal components. Neuropsychologia 1982;20:145-53.

[29] Dehaene S. Varieties of numerical abilities. Cognition 1992;44:1-42.

[30] Dehaene S. The organization of brain activations in number comparison: event-related potentials and the additive-factors method. Journal of Cognitive Neuroscience 1996;8:47-68.

[31] Dehaene S, Cohen L. Two mental calculation systems: a case study of severe acalculia with preserved approximation. Neuropsychologia 1991;29:1045-74.

[32] Dehaene S, Cohen L. Towards an anatomical and functional model of number processing. Mathematical Cognition 1995;1:83-120.

[33] Dehaene S, Cohen L. Levels of representation in number processing. In: Stemmer B, Whitaker HA, editors. Handbook of neurolinguistics. San Diego: Academic Press, 1998:331-41.

[34] Dehaene S, Dehaene-Lambertz G, Cohen L. Abstract representations of numbers in the animal and human brain. Trends-inNeurosciences 1998;21:355-61.

[35] Dehaene S, Dupoux E, Mehler J. Is numerical comparison digital: analogical and symbolic effects in two-digit number comparison. Journal of Experimental Psychology: Human Perception and Performance 1990;16:626-41.

[36] Dehaene S, Naccache L, Le Clec'H G, Koechlin E, Mueller M, Dehaene-Lambertz G, Van de Moortele PF, Le Bihan D. Imaging unconscious semantic priming. Nature 1998;395:597-600.

[37] Dehaene S, Tzourio N, Frak V, Raynaud L, Cohen L, Mehler J, Mazoyer B. Cerebral activations during number multiplication and comparison: a PET study. Neuropsychologia 1996;34:1097106.

[38] Delazer M, Girelli L, Semenza C, Denes G. Numerical skills and aphasia. Journal of the International Neuropsychological Society 1999;5:213-21.

[39] den Heyer K, Briand K. Priming single digit numbers: automatic spreading activation dissipates as a function of semantic distance. American Journal of Psychology 1986;99:315-40.

[40] Dimond S, Beaumont G. Use of two cerebral hemispheres to increase brain capacity. Nature 1971;232:270-1.

[41] Duncan EM, McFarland CE, Jr. Isolating the effects of symbolic distance and semantic congruity in comparative judgements: an additive factors analysis. Memory and Cognition 1980;8:612-22.
[42] Eviater Z. Language experience and right hemisphere tasks: the effects of scanning habits and multilingualism. Brain and Language 1997;58:157-73.

[43] Fias W, Brysbaert M, Geypens F, d'Ydewalle G. The importance of magnitude information in numerical processing: evidence from the SNARC effect. Mathematical Cognition 1996;2:95-110

[44] Gallistel CR, Gelman R. Preverbal and verbal counting and computation. Cognition 1992;44:43-74.

[45] Gazzaniga MS, Ledoux JE. The Integrated Mind. New York: Plenum, 1978.

[46] Gazzaniga MS, Smylie CE. Dissociation of language and cognition: a psychological profile of two disconnected right hemispheres. Brain 1984;107:145-53.

[47] Geffen G, Bradshaw JL, Wallace G. Interhemispheric effects on reaction time to verbal and nonverbal visual stimuli. Journal of Experimental Psychology 1971;87:415-22.

[48] Gordon HW, Carmon A. Transfer of dominance in speed of verbal response to visually presented stimuli from right to left hemisphere. Perceptual and Motor Skills 1976;42:1091-100.

[49] Hatta T. Visual field differences in semantic comparative judgements with digits and Kanji stimulus materials. Neuropsychologia 1983;21:669-78.

[50] Hatta T. Development of visual field advantage for digit and random shape recognition: comparison of cross-sectional and longitudinal studies. Neuropsychologia 1990;28:1053-62.

[51] Hatta T, Dimond SJ. Comparison of lateral differences for digit and random form recognition in Japanese and westerns. Journal of Experimental Psychology: Human Perception and Performance 1980;6:368-74.

[52] Hatta T, Tuji S. Interhemispheric integration of number stimulicomparison of Arabic with Kanji numerals. Cortex 1993;29:35964.

[53] Hatta T, Yoshizaki K. Effects of task demand on interhemispheric cooperation in mental addition task. Psychologia 1997;40:179-91.

[54] Hellige JB, Sergent J. Role of task factors in visual field asymmetries. Brain and Cognition 1986;5:200-22.

[55] Hines D, Satz P. Superiority of right visual half-fields in righthanders for recall of digits presented at varying rates. Neuropsychologia 1971;9:21-5.

[56] Hines D, Satz P. Cross-modal asymmetries in perception related to asymmetry in cerebral function. Neuropsychologia 1974;12:239-47.

[57] Hines D, Satz P, Schell B, Schmidlin S. Differential recall of digits in the left and right visual halffields under free or fixed order of recall. Neuropsychologia 1969;7:13-22.

[58] Hommel B. Event files: evidence for automatic integration of stimulus response episodes. Visual Cognition 1998;5:183-216.

[59] Katz AN. Cognitive arithmetic: evidence for right hemispheric mediation in an elementary component stage. Quarterly Journal of Experimental Psychology 1980;32:69-84.

[60] Kershner J, Thomae R, Callaway R. Nonverbal fixation control in young children induces a left-field advantage in digit recall. Neuropsychologia 1977;15:569-76.

[61] Kimura D. Right temporal lobe damage: perception of unfamiliar stimuli after damage. Archives-of-Neurology-Chicago 1963;8:264-71.

[62] Kimura D. Dual functional asymmetry of the brain in visual perception. Neuropsychologia 1966;4:275-85.

[63] Klein RM, McInnes J. Visual field differences in the processing of numerical stimuli. Brain and Cognition 1988;7:247-56.

[64] Koechlin E, Naccache L, Block E, Dehaene S. Primed numbers: exploring the modularity of numerical representations with masked and unmasked priming. Journal of Experimental Psychology: Human Perception and Performance 1999;25:1882-905. 
[65] Kosslyn SM, Koenig O, Barrett A, Cave CB, Tang J, Gabrieli JDE. Evidence for two types of spatial representations: Hemispheric specialisations for categorical and coordinate relations. Journal of Experimental Psychology: Human Perception and Performance 1989;15:723-35.

[66] Lambert AJ. Interhemispheric interaction in the split-brain. Neuropsychologia 1991;29:941-8.

[67] Lambert AJ. Attentional interaction in the split-brain: evidence from negative priming. Neuropsychologia 1993;31:313-24.

[68] Lorch RF, Myers JL. Regression analyses of repeated measures data in cognition research. Journal of Experimental Psychology: Learning, Memory and Cognition 1990;16:149-57.

[69] McGlone J, Davidson W. The relation between cerebral speech laterality and spatial ability with special reference to sex and hand preference. Neuropsychologia 1973;11:105-13.

[70] McCloskey M. Cognitive mechanisms in numerical processing: evidence from acquired dyscalculia. Cognition 1992;44:107-57.

[71] McCloskey M, Caramazza A, Basili A. Cognitive mechanisms in number processing and calculation: evidence from dyscalculia. Brain and Cognition 1985;4:171-96.

[72] Moscovitch M. Afferent and efferent models of visual perceptual asymmetries: theoretical and empirical implications. Neuropsychologia 1986;24:91-114.

[73] Moyer RS, Landauer TK. Time required for judgement of inequality. Nature 1967;215:1519-20.

[74] Ohtani Y. Visual field differences in an enumeration task of dots. Japanese Psychological Research 1985;27:206-14.

[75] Oldfield RC. The assessment and analysis of handedness: The Edinburgh inventory. Neuropsychologia 1971;9:97-113.

[76] Peereman R, Holender D. Relation entre taille physique et taille numerique dans la comparaison de chiffres ecrits alphabetique- ment et ideographiquement. Psychologica Belgica 1984;24:147264.

[77] Peereman R, Holender D. Visual-field differences for a numbernon-number classification of alphabetic and ideographic stimuli. The Quarterly Journal of Experimental Psychology 1985;36A:197-216.

[78] Pinel P, Le Clec'H G, Van de Moortele PF, Naccache L, Le Bilan D, Dehaene S. Event-related fMRI analysis of the cerebral circuit for number comparison. Neuroreport 1999;10:1473-9.

[79] Reynvoet B, Brysbaert M. Single and two-digit Arabic numerals address the same semantic number line. Cognition 1999;72:191201.

[80] Rosselli M, Ardila A. Calculation deficits in patients with right and left hemisphere damage. Neuropsychologia 1989;27:607-17.

[81] Seymour SE, Reuter-Lorenz PA, Gazzaniga MS. The disconnection syndrome: basic findings reaffirmed. Brain 1994;117:105-15.

[82] Teng EL, Sperry RW. Interhemispheric rivalry during simultaneous bilateral task presentation in commissurotomized patients. Cortex 1974;10:111-20.

[83] Umilta C, Rizzolatti G, Anzola GP, Luppino G, Porro C. Evidence of interhemispheric transmission in laterality effects. Neuropsychologia 1985;23:203-13.

[84] Warrington EK, James M. Tachistoscopic number estimation in patients with unilateral cerebral lesions. Journal-of-NeurologyNeurosurgery-and-Psychiatry 1967;30:468-74.

[85] Wu-Tian Z, Rui-Xiang P. The lateralization of hemispheric function in the recognition of Chinese characters. Neuropsychologia 1983;21:679-82.

[86] Young AW, Bion PJ. Hemispheric laterality effects in the enumeration of visually presented collections of dots by children. Neuropsychologia 1979;17:99-102. 